\title{
SLIDE: STREAMING AND LOAD-ADAPTIVE PERIODICITY ESTIMATION
}

\author{
Deepak S. Turaga, Michail Vlachos, Spiros Papadimitriou, and Philip S. Yu \\ IBM. T.J. Watson Research Center \\ 19 Skyline Dr, Hawthorne, NY
}

\begin{abstract}
We introduce a $C P U$-adaptive algorithm for spectrum estimation on continuous data. Our approach combines a fast and intelligent load-shedding scheme with a closed form incremental spectrum computation, which adapts gracefully to the available computational resources.
\end{abstract}

\section{INTRODUCTION}

Several emerging applications, including network traffic monitoring, financial data feeds, telemetry applications, medical data (e.g., ECGs), etc., contain streaming data arriving at high rates. Stream processing systems employ a collection of data analytic units which typically compete for available computational and memory resources. In such systems real-time response is crucial, therefore light-weight and efficient algorithms for processing and analyzing such data are highly desirable. Ultimately, however, the dominating constraint is the available system resources. Therefore, there is need for methods that can also gracefully degrade result accuracy based on CPU or memory availability; a good-enough answer is better than no answer at all.

Our goal is to efficiently estimate and update the periodogram of a signal, within a sliding time window. For fixedlength sequences, the periodogram can be estimated in $O(n \log n)$ time using the FFT. For dynamically updated sequences, the Momentary Fourier Transform (MFT) [1] can be employed to update the estimate over a sliding window. Recently, [2] proposes methods for periodicity estimation on streams, based on retaining the $k$ most significant Fourier coefficients. However, none of the streaming approaches address the issue of resource adaptation.

A simple approach for load adaptation would be to subsample the signal at regular intervals. However, this can lead to data aliasing and deteriorate the quality of the estimated periodogram. In contrast, our approach performs intelligent subsampling (see Fig. 2), based on a linear predictor which retains a sample only if its value cannot be predicted from its neighbors. This scheme allows us to efficiently make on-thefly decisions whether to discard a sample. Furthermore, we incorporate an estimator unit that adjusts the error tolerance of the predictor, based on available CPU time. Our scheme produces unevenly spaced samples. Therefore, we also introduce a closed-form Fourier approximation using uneven samples and we show how to update it incrementally. We call our load-adaptive methodology SLIDE (Streaming and Load-adaptive Periodicity Estimation). A schematic of our approach is provided on Fig. 1.

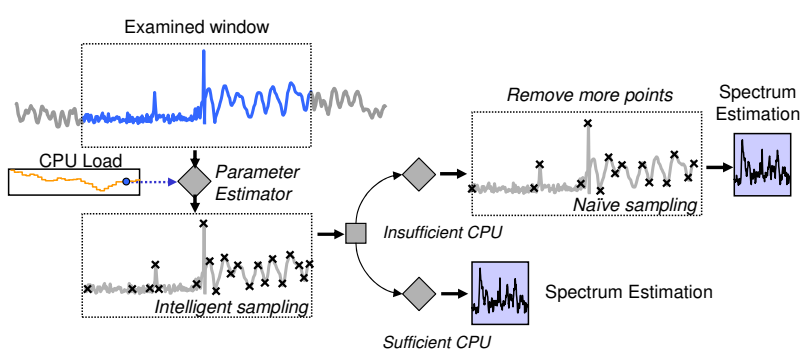

Fig. 1. Visual depiction of SLIDE.

Notation In the following, $x[k]$ is the $k$-th element ( $k \in$ $\mathbb{Z}^{+}$) of a discrete signal and $X[\mathrm{~m}]$ are its DFT coefficients. In this paper we use the periodogram of the signal as an estimator of the spectrum (and use the terms interchangeably). The notation $x\left[k_{i}\right]$ is used for the unevenly sampled signal, $k_{i}, i \in \mathbb{Z}^{+}$. Finally, we measure the complexity of our algorithms in terms of the number of additions (subtractions), multiplications and divisions (making the analysis independent of the underlying processor architecture). We label the complexity of a single multiplication as $\xi_{M u l}$, of a division as $\xi_{D i v}$ and of an addition/subtraction as $\xi_{S u b}$.

\section{LOAD-SHEDDING SCHEME}

We consider the typical problem of running spectral analysis where we slide a window across the temporal signal and incrementally update the signal's DFT (and the respective periodogram). As the data window slides by a fixed amount, we discard $n_{1}$ points from the beginning of the signal and add $n_{2}$ points to the end. However, if the available CPU cycles do not allow us to update the DFT using all the points, we can adaptively prune the set of added points to $\hat{n}_{2}$ using uneven sub-sampling to meet the CPU constraint while minimizing the impact on the accuracy of the updated DFT.

\subsection{Intelligent sampling via a linear predictor}

We determine if a sample can be retained based on whether it can be linearly predicted from its neighbors. In particular, 
for sample $k_{i}$ we compare the interpolated value $x^{i n t}\left[k_{i}\right]$ with the actual value $x\left[k_{i}\right]$, where

$$
x^{i n t}\left[k_{i}\right]=\frac{x\left[k_{i-1}\right]\left(k_{i+1}-k_{i}\right)+x\left[k_{i+1}\right]\left(k_{i}-k_{i-1}\right)}{k_{i+1}-k_{i-1}}
$$

and sample $k_{i-1}$ is the last retained sample before sample $k_{i}$ and sample $k_{i+1}$ is the immediately following sample. If $\left|x^{i n t}\left[k_{i}\right]-x\left[k_{i}\right]\right| \leq \frac{\Delta \times\left|x\left[k_{i}\right]\right|}{100}$ we can discard the sample $k_{i}$ otherwise we retain it. The parameter $\Delta$ is an adaptive threshold that determines the quality of the approximation.

The complexity of this algorithm for $M$ samples is :

$$
\xi^{s a m p}(M)=\left(2 \xi_{M u l}+4 \xi_{S u b}+\xi_{D i v}\right)(M-2)
$$

In Section 2.2 we discuss how to tune the threshold $\Delta$ in order to obtain the desired number of $N$ samples, out of the original $M$ samples.

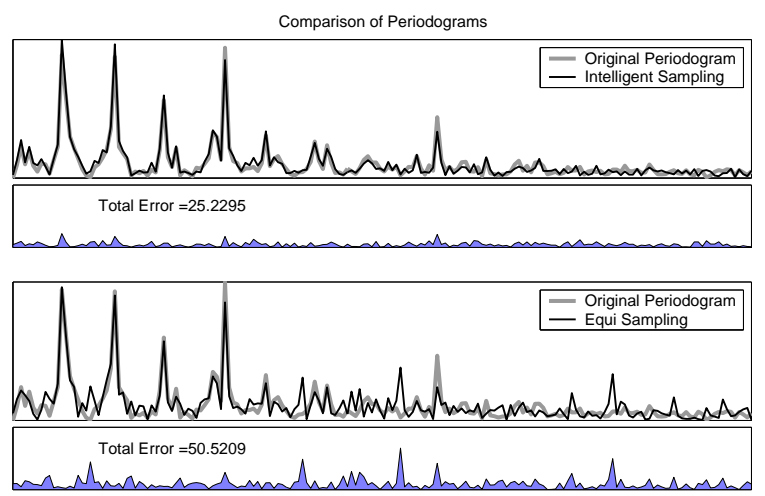

Fig. 2. Comparison of spectrum estimation errors for intelligent sampling and equi-sampling techniques.

Fig. 2 compares the spectrum estimates for a snapshot of a data stream, using the intelligent sampling method against a naïve equi-sampling technique, which samples data at a specified time interval. We execute our algorithm for a specific threshold and reduce the data points within a window from $M$ down to $N$. We estimate the resulting periodogram (see section 3) as well the periodogram derived by equi-sampling every $N / M$ points. Through the intelligent sampling one can provide higher quality reconstruction of the periodogram, because the important data stream features are retained.

In practice, our linear interpolation and thresholding schemes achieve good approximation of the signal, while requiring very simple calculations. It is also possible to derive a worst case bound of the cumulative error for each discarded sample. Let $\epsilon_{i}, i=1,2, \ldots$ denote the cumulative error for the first, second, etc, discarded samples after the last retained one. Let $i=N$ be the last sample we chose to discard. Our decision at that point is based on the instantaneous error $\delta_{N}:=x[N]-x^{i n t}[N]$, i.e., we discard $x[N]$ if $\left|\delta_{N}\right| \leq$ $\Delta|x[N]| \leq \Delta \max _{i=1}^{N}|x[i]|$. By triangle similarity, it is easy to see (Figure 3 ) that the worst case contribution to the cumulative error of all previous discarded samples is

$$
i \Delta|x[N]| / N \leq i \Delta \max _{j=1}^{N}|x[j]| / N, \text { for } i=1, \ldots, N-1
$$

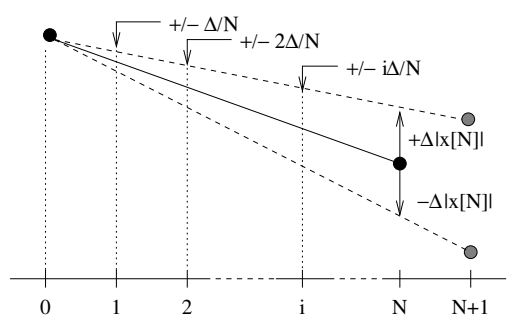

Fig. 3. Contribution of instantaneous error at $t=N$ to cumulative error of previously discarded points.

Summing these up we get

$$
\begin{aligned}
\left|\epsilon_{i}\right| & \leq(\Delta+i \Delta(1 /(i+1)+\cdots+1 / N)) \max _{i}|x[i]| \\
& \approx(\Delta+i \Delta(\ln N-\ln i)) \max _{i}|x[i]| \\
& =\Delta(1+i \ln (N / i)) \max _{i}|x[i]|
\end{aligned}
$$

where we use the approximation $\sum_{i=1}^{N} \frac{1}{i} \approx \ln N$ for the harmonic series. This is maximized for $i=N \max _{i}|x[i]| / e$ and the maximum (over all discarded samples) of the worst case cumulative error is $\Delta(1+N / e)$. Since the sequence is variance scaled, $\max _{i}|x[i]|$ is typically small and can be ignored. This corresponds to a sequence such that $x[t]=$ $\frac{t}{t-1}(x[t-1] \pm \Delta)+x[0]$, for $t=2,3, \ldots$.
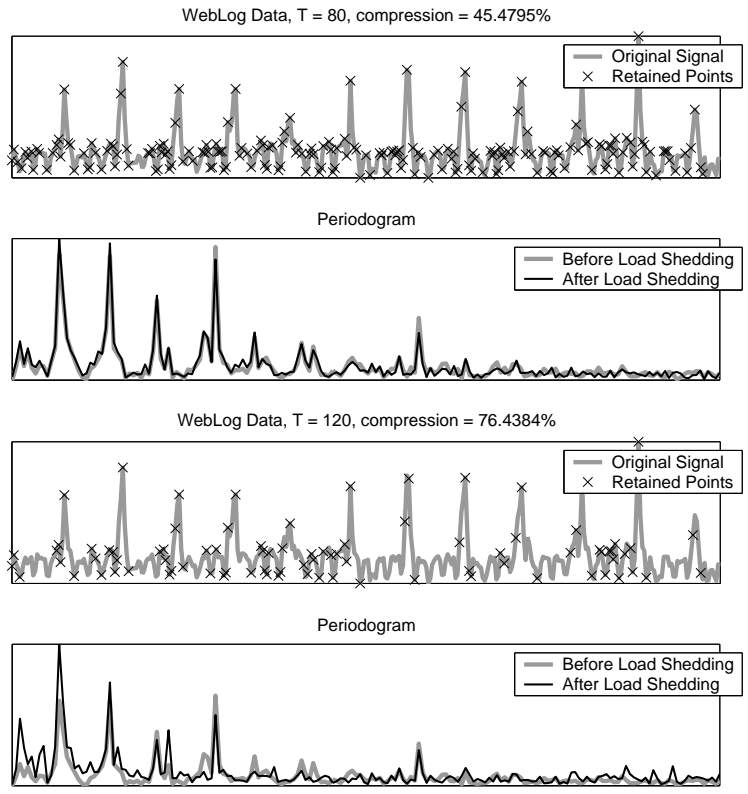

Fig. 4. Spectrum approximation for different threshold values

\subsection{Threshold Estimator}

Our goal is to predict the threshold $\Delta$ that will produce a desired number of uneven samples $N$ during the next time window of length $M$ in the future. The estimation of $\Delta$ is based on the behaviour of the signal during the past window of same size. Formally, let $k_{c}$ be the current sample at the time we 
wish to readjust for available resources and let $x[k: k+M-$ $1]:=(x[k], x[k+1], \ldots, x[k+M-1]) \in \mathbb{R}^{M}$ be a window of $M$ even samples. Then, we want a mapping $p: \mathbb{R}^{M} \times \mathbb{N} \mapsto \mathbb{R}$ giving us the threshold $\Delta=p\left(x\left[k_{c}-M+1: k_{c}\right], N\right)$.

However, the domain $\mathbb{R}^{M} \times \mathbb{N}$ of $p$ has excessively high dimensionality. Our practical solution is to summarize $x[k$ : $k+M-1$ ] by a small set of features which capture the "irregularity" of the signal within that window. Formally, let $f: \mathbb{R}^{M} \mapsto \mathcal{F}$ be a mapping from the actual window to a sufficiently small feature set $\mathcal{F} \subseteq \mathbb{R}^{d}$, where $d \ll M$. The threshold estimator we use in practice is a mapping $\hat{p}: \mathcal{F} \times \mathbb{N} \mapsto \mathbb{R}$. The interpolation threshold we choose is $\hat{\Delta}=\hat{p}\left(f\left(x\left[k_{c}-\right.\right.\right.$ $\left.\left.M+1: k_{c}\right], N\right)$.

The feature we use is a per-band variance $f_{\text {freq }}$. If $X[\mathrm{~m}]$, $0 \leq m \leq M-1$ are the DFT coefficients of $x[k: k+M-1]$, then we divide the frequencies into $B$ bands of equal width to obtain

$$
f_{\text {freq }}(x[k: k+M-1]):=\left(v_{0}, v_{1}, \ldots, v_{B-1}\right) \in \mathbb{R}^{B},
$$

where $v_{j}:=\sum_{m=j(M-1) / B+1}^{(j+1)(M-1) / B} X^{2}[m], 0 \leq j \leq B-1$. Note that the DC coefficient $X[0]$ is omitted from $f_{\text {freq. }}$. When $B=1$, then $v_{0}=\operatorname{Var}(x[k: k+M-1])$. The per-band variance provides a finer characterization of the irregularities than just the variance. Small number of bands $B$ (e.g., $B=2$ or $B=4$ ) provide good estimators without increasing the space complexity. These features can be incrementally maintained over a sliding window of size $M$.

The next step is how we compute the estimate $\hat{p}$. To that end, we use a training set $\mathcal{W}:=\left\{W_{j} \mid W_{j}=x\left[i_{j}\right.\right.$ : $\left.\left.i_{j}+M-1\right], 1 \leq j \leq w\right\}$, consisting of $w$ windows. We run our algorithm on each window for several different thresholds and get value of $N$ for each of them. This produces a training set $\mathcal{T}$ of examples $S_{l} \in \mathcal{F} \times \mathbb{N} \times \mathbb{R}$, i.e., $\mathcal{T}:=\left\{S_{l} \mid\right.$ $\left.S_{l}=\left(f\left(W_{j_{l}}\right), N_{l}, \Delta_{l}\right), W_{j_{l}} \in \mathcal{W}\right\}$. We use the subscript $l$ to identify elements of $\mathcal{T}(1 \leq l \leq|\mathcal{T}|)$.

We employ a $k$-NN ( $k$ nearest-neighbor) interpolation scheme to estimate $\hat{p}$, where the neighbor distance is computed only with respect to the features $f\left(W_{j_{l}}\right)$ and the number of retained samples $N_{l}$. More specifically, if $W:=x\left[k_{c}-M+1\right.$ : $\left.k_{c}\right]$, then $\operatorname{kNN}(f(W), N)$ is the set of $k$ elements $S_{l} \in \mathcal{T}$ with the smallest distances $\|\left(f\left(W_{j_{l}}, N_{l}\right)-(f(W), N) \|\right.$ from $(f(W), N)$, among all elements of $\mathcal{T}$. Then

$$
\hat{\Delta}=\hat{p}_{\mathrm{kNN}}(f(W), N):=\frac{1}{k} \sum_{S_{l} \in \mathrm{kNN}(f(W), N)} \Delta_{l} .
$$

Additionally, $\mathcal{T}$ can also be incrementally refined over time, by incorporating examples that haven't been encountered during the training phase. This can minimize the potential errors of the threshold estimator, even under significant changes in the stream pattern.

\section{SPECTRUM ESTIMATION FOR UNEVENLY SAMPLED SIGNALS}

Given $N$ uneven samples $x\left[k_{n}\right], 0 \leq n \leq N-1$, we estimate the periodogram as follows. Conceptually, we first use linear interpolation (as for the sub-sampling) to reconstruct the evenly sampled signal $x[k]$ and then estimate the DFT $X[m]$ from it. However, we do not actually need to perform the interpolation and instead, we can directly derive closed form expressions, as in [3], for the DFT of $x\left[k_{i}\right]$ as:

$$
X[m]=\sum_{n=1}^{N-1} X_{n}[m]
$$

where, for $m=1, \ldots, M-1$,

$$
\begin{aligned}
& X_{n}[m]=\frac{1}{\left(k_{n}-k_{n-1}\right)\left(\frac{2 \pi m}{M}\right)^{2}}\left[\left(x\left[k_{n-1}\right]-x\left[k_{n}\right]\right) .\right. \\
& \cdot\left(e^{-j \frac{2 \pi m k_{n}-1}{M}}-e^{-j \frac{2 \pi m k_{n}}{M}}\right)+ \\
& \left.+j \frac{2 \pi m}{M}\left(x\left[k_{n}\right] e^{-j \frac{2 \pi m k_{n}}{M}}-x\left[k_{n-1}\right] e^{-j \frac{2 \pi m k_{n}-1}{M}}\right)\right],
\end{aligned}
$$

and for $m=0$,

$$
X_{n}[0]=\frac{1}{2}\left(x\left[k_{n-1}\right]+x\left[t_{n}\right]\right)\left(k_{n}-k_{n-1}\right) .
$$

Note that, while $x\left[k_{i}\right]$ has $N$ samples, the DFT has at least $M=k_{N-1}-k_{0}$ samples to avoid time domain aliasing.

\subsection{Incremental Spectrum Estimation for Streaming Data}

A significant benefit of equation (3) is that the DFT for unevenly sampled signals can be evaluated incrementally. Hence, if we shift the window (of size $M$ ) such that $n_{1}$ points are discarded, and $n_{2}$ new points are added (i.e. we have $N+n_{2}-n_{1}$ points), then the DFT of the signal may be updated as:

$$
X^{\text {new }}[m]=X^{\text {old }}[m]-\sum_{n=1}^{n_{1}} X_{n}[m]+\sum_{n=N}^{N+n_{2}-1} X_{n}[m]
$$

We now examine the complexity of this update. Similar to prior analyses of FFT complexity, we do not consider the complexity of computing $e^{\frac{j 2 \pi m k_{n}}{M}}$ (and the intermediate value $\frac{2 \pi m k_{n}}{M}$ ). The complexity of computing $X_{n}[m]$ is

$$
\begin{aligned}
\hat{\xi}_{n z} & =6 \xi_{\text {Mul }}+5 \xi_{\text {Sub }}+\xi_{\text {Div }}, & & m=1, \ldots, M-1, \\
\hat{\xi}_{z} & =2 \xi_{\text {Mul }}+2 \xi_{\text {Sub }}, & & m=0 .
\end{aligned}
$$

If we define $\hat{\xi}_{\text {all }}=(M-1) \hat{\xi}_{n z}+\hat{\xi}_{z}$, the total update complexity is

$$
\xi^{u p}\left(M, n_{1}, n_{2}\right)=\left(n_{1}+n_{2}\right)\left[\hat{\xi}_{\text {all }}+M \xi_{\text {Sub }}\right]+2 M \xi_{\text {Sub }}
$$

\subsection{Complexity Reduction with Sub-sampling}

When the window shifts, we cannot adapt the number of points discarded $\left(n_{1}\right)$, however we can reduce the number of new points added $\left(n_{2}\right)$ through intelligent sub-sampling. Consider that the sub-sampling results in $\hat{n}_{2}$ samples $\left(\hat{n}_{2} \leq n_{2}\right)$. Comparing equations (9) and (2) we realize that the overall complexity of updating the spectrum estimate is reduced when:

$$
\xi^{u p}\left(M, n_{1}, n_{2}\right) \geq \xi^{u p}\left(M, n_{1}, \hat{n}_{2}\right)+\xi^{s a m p}\left(n_{2}\right)
$$

Consider a simple case when $\hat{n}_{2}=n_{2}-1$, i.e. sub-sampling discards one sample. The sub-sampling complexity is $\left(2 \xi_{M u l}+\right.$ 


\begin{tabular}{|c|c|c|c|c|c|}
\hline Dataset & Threshold & Window Compression (\%) & Error Equi-Sampling & Error Intelligent & Improvement (\%) \\
\hline \multirow[t]{3}{*}{ ECG } & 20 & 80.96 & 1627.55 & 450.79 & 72.30 \\
\hline & 60 & 91.40 & 2434.59 & 1326.23 & 45.52 \\
\hline & 100 & 95.79 & 2934.84 & 2171.04 & 26.02 \\
\hline \multirow[t]{3}{*}{ EEG } & 20 & 6.73 & 79.79 & 2.76 & 96.53 \\
\hline & 60 & 18.45 & 202.03 & 33.10 & 83.61 \\
\hline & 100 & 32.81 & 221.16 & 105.99 & 52.07 \\
\hline \multirow[t]{3}{*}{ RTT } & 20 & 35.90 & 147.76 & 26.68 & 81.94 \\
\hline & 60 & 60.69 & 174.24 & 81.21 & 53.38 \\
\hline & 100 & 75.55 & 210.69 & 123.98 & 41.15 \\
\hline \multirow[t]{3}{*}{ WebTrace } & 20 & 13.97 & 22.08 & 4.04 & 81.70 \\
\hline & 60 & 37.26 & 46.29 & 18.98 & 58.99 \\
\hline & 100 & 61.36 & 52.31 & 47.52 & 9.15 \\
\hline
\end{tabular}

Table 1. Accuracy of periodogram using Intelligent and Equi-Sampling

$\left.4 \xi_{S u b}+\xi_{D i v}\right)\left(n_{2}-2\right)$ while the decrease in the update complexity is $(M-1)\left(6 \xi_{M u l}+5 \xi_{S u b}+\xi_{D i v}\right)+\left(2 \xi_{M u l}+2 \xi_{S u b}\right)+$ $M \xi_{S u b}$. Clearly, since $\hat{n}_{2}<n_{2} \leq M$, we can easily realize that the reduction in update complexity far outweighs the subsampling complexity. In general, equation (10) is always true when the sub-sampling reduces the number of samples (i.e when $\hat{n}_{2}<n_{2}$ ). If, at a certain time, the CPU imposes a computation constraint of $\xi^{\text {limit }}$, and $\xi^{u p}\left(M, n_{1}, n_{2}\right)>\xi^{\text {limit }}$ we can determine the optimal number of samples to retain $\hat{n}_{2}$, as:

$\hat{n}_{2} \leq \frac{\xi^{l i m i t}-\xi^{s a m p}\left(n_{2}\right)-2 M \xi_{\text {Sub }}}{(M-1)\left(6 \xi_{M u l}+5 \xi_{\text {Sub }}+\xi_{\text {Div }}\right)+\left(2 \xi_{M u l}+2 \xi_{\text {Sub }}\right)+M \xi_{\text {Sub }}}-n_{1}$

We can achieve this by tuning the threshold $\Delta$ based on the algorithm described in Section 2.2.

\section{EXPERIMENTS}

We examine two parameters of the resource-adaptive spectrum estimation: (1) The accuracy of the approximated periodogram, (2) The CPU adaptiveness of our technique, which depends on the quality of the threshold estimator. We measure the periodogram error on various datasets, and for different threshold values of the linear predictor. For a given threshold, a data window of length $M$ will be reduced to $N$ samples. We compare the quality of the approximated periodogram against a rudimentary approach that performs equisampling every $N / M$ points. The results are given in Table 1 and clearly indicate that the proposed load-shedding scheme leads to high quality spectrum estimates. The reduction in the estimation error compared to equi-sampling, ranges from $10 \%$ to more than $90 \%$.
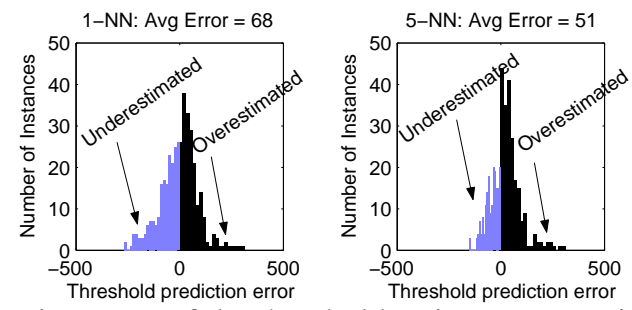

Fig. 5. Histogram of the threshold estimator error, indicating the cases of overestimated and underestimated threshold
Next, we measure the accuracy of the threshold estimator using streaming automotive measurements. Periodic analysis is an indispensable tool in automotive industry, because changes in the vibration pattern of an engine can provide valid indications of an upcoming failure. We feed our adaptive spectrum estimation unit with a synthetic CPU load, which gives enough time to process samples in the range of $[|w| / 20 \ldots|w|]$ (where $|w|=1024$ is the window length).

The threshold estimator is first trained on a small subset of the data. We use $B=2$ per-band variance features. The accuracy of the estimator is evaluated on streaming data and for each sliding data window the estimator error is captured as $|\hat{N}-N|$, where $\hat{N}$ is the desirable number of retained samples given the current CPU load and $N$ is the actual one (based on the selected threshold $\Delta$ ).

We evaluate two versions of the estimator; one that uses 1-NN interpolation and another with 5-NN interpolation. Histograms of the approximation error are provided on Fig. 5. The empirical error distribution indicates that for the majority of cases the estimation error is small. On the figure we plot separately the cases of overestimated and the underestimated thresholds. For 5-NN interpolation the instances of overestimated threshold (fewer remaining points than expected) are higher than the underestimated. This is more desirable since we don't have to resort to the additional phase of random resampling.

\section{CONCLUSION}

We presented a spectrum estimation method that can adapt its quality based on the CPU load. Compared to equi-sampling, our intelligent load-shedding scheme can introduce improvements on the spectrum estimation ranging from $10 \%$ to $90 \%$.

\section{REFERENCES}

[1] A. Papoulis. Signal Analysis. McGraw-Hill, 1977.

[2] A.C. Gilbert, S. Guha, P. Indyk, S. Muthukrishnan, and M. Strauss. Near-optimal Sparse Fourier Representations via Sampling. In STOC, pages 152-161, 2002.

[3] P. Castiglioni, M.D. Rienzo, and H. Yosh. A Computationally Efficient Algorithm for Online Spectral Analysis of Beat-to-Beat Signals. In Computers in Cardiology:29, 417:420, 2002. 